\title{
Editorial: Organ Modification for Edible Parts of Horticultural Crops
}

\author{
Yuke $\mathrm{He}^{1 *}$, Guusje Bonnema ${ }^{2}$, Han Xiao ${ }^{1}$ and Yunde $\mathrm{Zhao}^{3}$ \\ ${ }^{1}$ National Key Laboratory of Plant Molecular Genetics, Shanghai Institute of Plant Physiology and Ecology, Chinese Academy \\ of Sciences, Shanghai, China, ${ }^{2}$ Plant Breeding, Wageningen University and Research, Wageningen, Netherlands, ${ }^{3}$ Section \\ of Cell and Developmental Biology, University of California, San Diego, San Diego, CA, United States
}

Keywords: morphological modification, corm, bulb, curd, fleshy fruit, fleshy root, leafy head, tuber

\section{Editorial on the Research Topic}

\section{Organ Modification for Edible Parts of Horticultural Crops}

Plant development refers to various cellular processes that govern the morphogenesis of root, stem, leaf, flower, fruit, and seed. Plant development is regulated by genetic, epigenetic, and environmental factors. Developmental processes includes the determination, initiation, differentiation, and expression of different types of primordia on shoot/root apical meristems, and involve the establishment of polarity, cell division and differentiation of cell and tissue, phase transition, and hormone responses. Plant organs such as leaf, stem, root, flower, and fruit plays roles in water and mineral absorption, transportation, photosynthesis, pollination, fertilization, and other physiological processes. However, many horticultural crops often display enlarged organs to develop into the edible products: leafy head, bulb, tuber, fleshy stem, corm, fleshy root, root tuber, curds, and fleshy fruits. Unlike the grains of many field crops that mainly provide starch, the modified organs in horticultural crops store vitamins, secondary metabolites, minerals, and dietary fiber that are important for human health. Their commercial quality mainly depends on the diversity in size, shape, surface features, and texture. It is very interesting to know how these plant organs were selected and domesticated to become the edible parts of horticultural crops. Recently, molecular biology technology have been applied to address the key scientific questions and research directions with regard to morphological modifications of leaf, stem, root, flower, and fruit. For example, several miRNAs have been identified to play essential roles in determination of size, shape, and timing of leafy heads (Mao et al., 2014; Wang et al., 2014; Ren et al., 2018).

The chapters address genetic and molecular basis of morphological modifications of plant organs in horticultural crops. The focused topic issues cover dynamic phenotyping of the modified organs: leafy head, bulb, tuber, fleshy stem, corm, fleshy root, root tuber, curd, and fleshy fruit during plant development, genetic variation of modified organs, functional analysis of genes of organ-related traits that control size, shape, weight, texture, color and flavor of the modified organs, regulation of non-coding RNAs controlling morphological modification, effects of abiotic stress molecular mechanisms underlying metamorphosis of plant organs.

Genetic basis of leafy heads and bulbs is a critical topic. Many vegetable crops develop into certain types of leaf curvature required for high yield and quality. Recent research has revealed that miRNAs in Brassica crops regulate the timing and shape of leafy heads (Mao et al., 2014; Wang et al., 2014). Several papers in this issues described the origin, diversity, and development of leafy heads. Three papers ("Genetic analysis of Chinese cabbage reveals correlation between rosette leaf and leafy head variation" by Sun et al.; "Characterization of non-heading mutation 
in heading Chinese cabbage (Brassica rapa L. ssp. pekinensis)" by Li et al.; and "Transcription coactivator ANGUSTIFOLIA3 (AN3) regulates leafy head formation in Chinese Cabbage" by $\mathrm{Yu}$ et al.). These papers described the phenotyping, DNA resequencing and gene function identification of Chinese cabbage. Modification of flower organ is important for function transformation of edible organs. Two papers are related to flower organ modifications ("Differential gene expression caused by the $\mathrm{F}$ and $\mathrm{M}$ loci provides insight into ethylene-mediated female flower differentiation in cucumber" by Pan et al. and "defective apetala2 genes lead to sepal modification in Brassica crops" by Zhang et al.). Shape, size, color, and flavor of fleshy fruits are genetically related to leaf features. One paper "Chemical composition and crystal morphology of epicuticular wax in mature fruits of 35 pear (Pyrus spp.) cultivars" by Wu et al. "discloses crystal morphology of "epicuticular wax in mature fruits;"” and another paper "Functional dissection of Auxin Response Factors in regulating tomato leaf shape development" by $\mathrm{Wu}$ et al. provide the evidences that auxin response factors regulate tomato leaf shape and fruit development. The paper "Plant organ shapes are regulated by protein interactions and associations with microtubules" addressed the importance of microtubules to formation of organ shape (Lazzaro et al.). Finally, this book contains eight papers from scientists working in institutions from different countries. Thus, we can claim that this book, with its multiple scientific voices, provides also a contribution to understanding

\section{REFERENCES}

Mao, F., Wu, F., Yu, X., Bai, J., Zhong, W., and He, Y. (2014). microRNA319atargeted Brassica rapa ssp. pekinensis TCP genes modulate head shape in Chinese cabbage by differential cell division arrest in leaf regions. Plant Physiol. 164, 710-720. doi: 10.1104/pp.113.228007

Ren, W., Wang, H., Bai, J., Wu, F., and He, Y. (2018). Association of microRNAs with types of leaf curvature in Brassica rapa. Front. Plant Sci. 9:73. doi: $10.3389 /$ fpls.2018.00073

Wang, Y., Wu, F., Bai, J., and He, Y. (2014). BrpSPL9 (Brassica rapa ssp. pekinensis SPL9) controls the earliness of heading time in Chinese cabbage. Plant Biotechnol. J. 12, 312-321. doi: 10.1111/pbi.12138 genetic basis and molecular mechanisms underlying organ modification.

\section{AUTHOR CONTRIBUTIONS}

All authors listed have made a substantial, direct and intellectual contribution to the work, and approved it for publication.

\section{FUNDING}

This work was supported by grants from the National Key Research and Development Program of China (Grant No.2016YFD0101900), Natural Science Foundation of China (Grant Nos. 31771442 and 31571261), The National Key Research and Development Program of China (2016YFD0100500), Shanghai Agriculture Applied Technology Development Program, China (Grant No. Z20160109), and Shanghai Agriculture Applied Technology Development Program, China (Grant No.G2015060107), Dutch Royal Academy of Sciences China Exchange Program (Grant No. 530-4CDP08), the National Key R\&D of China (Grant No. 2017YFD0101802), the National Natural Science Foundation of China (Grant No. 31672151), International Cooperation project in the Science and Technology Support Program of Hebei (Grant No. 17396315D), Hundred Innovative Talent Program of Hebei (Grant No. SLRC2017040), the Natural Science Foundation of Hebei (C2016204170), and the Science and Technology Support Program of Hebei (16226304D-2).
Conflict of Interest Statement: The authors declare that the research was conducted in the absence of any commercial or financial relationships that could be construed as a potential conflict of interest.

Copyright (c) 2019 He, Bonnema, Xiao and Zhao. This is an open-access article distributed under the terms of the Creative Commons Attribution License (CC BY). The use, distribution or reproduction in other forums is permitted, provided the original author(s) and the copyright owner(s) are credited and that the original publication in this journal is cited, in accordance with accepted academic practice. No use, distribution or reproduction is permitted which does not comply with these terms. 